\title{
'In reference to HIV, what does 'undetectable mean to you?': Results of a small survey at an HIV testing site in San Francisco
}

\author{
Jason Johnson Peretz
}

\begin{abstract}
Objectives: 'Undetectable', a term describing HIV viral load at the level of non-transmissibility, appears to be used as a stigma-reducing term on dating apps and is predictive of condomless sex. Anecdotal evidence about the meaning of the term suggests that a mix of both accurate data and misinformation can abound. We sought to uncover what range of meanings the term 'undetectable' holds for a cohort of HIV testers at a free HIV testing facility along mid-Market St, San Francisco.

Design: A four question survey with additional demographic questions was administered to 130 individuals at the testing site. The resulting answers were then coded as 'informed', 'uninformed', or 'misinformed' and correlated with demographic measures, including residential zip code of the respondent.

Results: Residents in the Castro district were most likely to associate 'undetectable' status with medication adherence. The most misinformed zip code with respect to the meaning and implications of the term 'undetectable' was the Tenderloin, which corresponds to the San Francisco zip code with the lowest rates of HIV suppression. Compared to MSMs, heterosexuals were the most uninformed; while racially, Asians were the most misinformed and Blacks the least misinformed. Older men seem most cautious about using 'undetectable' as a risk-reduction strategy.

Significance: The study highlights the degree to which certain neighborhoods and demographics have assimilated the concept of 'undetectable' and how viral suppression is maintained, while pinpointing neighborhoods for additional outreach to correct identified misinformation.
\end{abstract}

Keywords: undetectable, HIV, viral suppression, ARV, adherence, San Francisco, MSM, Tenderloin, Castro

\section{Introduction and Background}

Antiretroviral therapy (ART) has shown great success in reducing HIV viral load in the blood, leading not only to healthier lives, but also to reduced transmission of HIV through sexual contact. Advances in the minimum detectable units per millilitre of blood, has meant the defined threshold for having an 'undetectable' viral load has steadily lowered since the late 1990s, from 350 units/ mL two decades ago to 40 units/ mL or less in 2016. [2] An 'undetectable' viral load is associated with non-transmissibility of the virus during unprotected sexual encounters between serodiscordant couples. $[1,2]$ Lowering community viral load has been shown to decrease transmission of HIV. [9, 10]

The term 'undetectable' has been increasingly adopted by persons living with HIV as indicative of their level of ART adherence. 'Undetectable' appears to be used as a stigma-reducing term on hook-up apps. [6, 7] Together with the widespread uptake of PrEP, both 'negative on PrEP' and 'undetectable' are 'treatment as prevention' identity-based terms deployed during sexual encounters to assess whether a sex will be initiated, and if so, whether condoms will or will not be used. $[3,4,5]$

In this environment, a mix of both accurate data and misinformation can abound. Anecdotal evidence from counselors suggest that clients are uncertain what the term 'undetectable' actually means. Clients coming in for testing and acquaintances, including men on hook-up apps as Grindr, who know a counselor's association with HIV testing and outreach, ask them what 'undetectable' implies, and what follow-up questions should be asked when people say they are 'undetectable'. [6] Additionally, studies conducted at a local, urban safety-net clinic, and conversations overheard on public transportation by fellow researchers, have indicated highly variable understandings of the term 'undetectable' even among persons living with HIV. [7]

Neighborhoods within cities are differently affected by the burden of infectious disease, and HIV is no exception. In San Francisco, surveillance includes data on rates of new infections and degree of viral suppression by neighborhood. $[8,12]$ Together with data from other viral load tests for people engaged in care, these data sets can be used to determine approximate community viral load.

Understanding the range of informed, misinformed, and uninformed understandings of health related 
terminology is part and parcel of public health outreach. In the context of San Francisco and California's 'Getting to Zero' campaign, assessing what people understand by the term 'undetectable' is essential to pinpointing what messaging is going through, which communities are picking up on it, and where the messaging is missing the mark.

This study aimed to assess the range and depth of knowledge about this term among clients who tested at a local HIV testing site in November and December 2016. We strove to incorporate a geographic identifier into our study to assess the degree of information and misinformation on a neighborhood by neighborhood bases, using zip codes as a proxy.

\section{Materials and Methods}

Over a two month period, we deployed a survey consisting of a brief initial paragraph explaining the purpose of the study, four questions, and a line for demographic information. The survey was self-administered at a testing site in San Francisco serving a demographic of primarily men who have sex with men. Although MSM are the primary demographic, this site administers a rapid p24 and HIV antibody test for anyone who comes in for HIV testing.

A pilot survey of three questions was developed and given to several individuals to determine whether the questions were read in the way intended. Taking account of pilot respondents' feedback, the three questions were finalised as follows:

1. With reference to HIV, what does 'undetectable' mean to you?

2. How does someone know if they are undetectable?

3. Once someone has been told they are undetectable, for how long does that person remain undetectable?

After discussing the deployment with supervisors at the testing site, a fourth question, asking how we could encourage clients to test every three to six months was added. Demographic information included age, race, gender, relationship status, HIV status, and zip code. Zip code was included as a proxy for neighborhood of residence. We wanted to see whether information saturation was more present in some neighbourhoods compared to others. Sexual orientation was not requested in the demographic section because the survey was attached to a standardized testing intake form which assessed sexual history. Sexual orientation data was derived from responses to questions about sexual behaviour, rather than from self-identification terms like 'gay', 'queer', 'straight', etc.

After approval by the directors of the HIV Counselling and Testing program at the survey site, we added the survey to the usual intake forms for clients to fill out in the waiting area when they came to get tested. These forms were handed to the client by front desk staff. The clients gave all completed forms to whichever California state certified HIV test counselor guided them through the testing process that day. Counselors were both male and female. The completed surveys were kept with the full records until collected for recording and coding.

Although the consent forms for testing are confidential, the survey sheet itself was anonymous. For this reason, and because SF DPH recommends sexually active MSMs get tested for STIs and HIV every three to six months, the study was limited to two months in order to decrease likelihood of repeat participants.

The responses were recorded in a spreadsheet with a column to record each question's responses and all demographic information, for ease of sorting. Responses to each question were coded and grouped according to content and theme. Initial groupings were based on separating out responses indicating uncertainty or lack of knowledge from offered answers. Offered answers were then grouped according to theme (e.g. 'medication', 'viral load test') and degree of information or misinformation the response indicated. Ultimately, responses were classed simply as 'informed', 'uninformed', and 'misinformed'. Thematic groupings were then analysed for possible demographic trends.

\section{Results}

\section{Demographics}

Of 137 surveys returned to us, 130 contained at least one response. We were able to collect full demographic information on 122 individuals. Men accounted for 112 respondents, women for 9 respondents (6 cis-female, 3 
transfemale). Seven participants did not answer the question on gender, and one respondent listed gender as 'unknown'. Most women were white (4 cis-gender, 1 transgender), followed by two Black women (1 cis-, one trans-) and two Latina women (1 trans, 1 unknown). Only one Asian woman tested (cis-gender).

The average age of respondents was 35, with 32 being the median. The age range was between 16 years and 67 years of age. The most common age was 28 (9 individuals). White people had the greatest range of ages (18 67), followed by Blacks (19 - 55), Latinos (19 - 46), and Asians (16 - 38).

In terms of race, nine people did not respond. Of those who did indicate race, $49 \%$ identified as White, $20 \%$ as Asian, $13 \%$ as Latino or Hispanic, $12 \%$ as Black, and $6 \%$ as mixed race, which included black/white, asian/white, and hispanic/ white, information volunteered by respondents.

Sexuality, as determined by reference to the attached intake form, included 78 MSM (white [38], asian [14], latino [10], black [8]), 18 heterosexual men (asian [7], white [6], black, latino [2]), 7 heterosexual women (i.e. the entire cis-female sample), and 3 bisexual men (white [2], asian [1]). Information on sexual orientation was not available for 19 people, as their intake forms had become detached from the surveys and could not be traced back on the basis of other demographic information (e.g. zip code, age, race).

Table 1: Survey Respondent Demographics

\begin{tabular}{|c|c|c|c|c|c|}
\hline & $\mathbf{N}=$ Known & $\mathbf{N}=$ Unknown & & $\mathbf{N}=$ Known & $\mathbf{N}=$ Unknown \\
\hline Administered & 137 & 0 & Race & 113 & 9 \\
\hline Response $>0$ & 130 & 7 & Asian & 24 & \\
\hline $\begin{array}{l}\text { Contains demo } \\
\text { data }\end{array}$ & 122 & 8 & Black & 15 & \\
\hline Gender & 121 & 9 & Latino & 16 & \\
\hline Male & 112 & & Multi-racial & 7 & \\
\hline Female & 9 & & White & 60 & \\
\hline Cis-female & 6 & & $\begin{array}{l}\text { Relationship } \\
\text { Status }\end{array}$ & 101 & 21 \\
\hline Trans-female & 3 & & Single & 77 & \\
\hline Asian & 1 & & Married & 10 & \\
\hline Black & 2 & & Partnered & 14 & \\
\hline Latina & 2 & & Sexual behavior & 103 & 19 \\
\hline White & 4 & & MSM & 78 & \\
\hline Age & 116 & 8 & MSF & 18 & \\
\hline Mean & 35 & & MSM\&F & 3 & \\
\hline Median & 32 & & FSM & 7 & \\
\hline Mode & 28 & & Zip Codes & 99 & 23 \\
\hline Range & $16-67$ & & San Francisco & 93 & \\
\hline Asian & $16-38$ & & Other California & 15 & \\
\hline Black & $19-55$ & & Out of State & 1 & \\
\hline Latino & $19-46$ & & Out of Country & 1 & \\
\hline White & $18-67$ & & & & \\
\hline
\end{tabular}


Most respondents were single (77). Married respondents account for 10 surveys, with an additional 14 reporting they were partnered or in a relationship. Two people specified their relationship status as 'open', two as 'couple' and two as 'boyfriend'. One respondent specified he has a boyfriend who is HIV+ and undetectable.

According to zip codes, most respondents lived in the Castro ( $\mathrm{n}=14$, or $11 \%$ of sample), followed by the Inner Mission/ Bernal Heights $(\mathrm{n}=11=9 \%)$. SoMa and the Tenderloin/ Hayes Valley neighbourhoods accounted for 9 responses each $(=7 \%)$, while Twin Peaks/ Glen Park saw 8 respondents $(=7 \%)$.

Two HIV+ individuals responded to the survey, perhaps because they came in to support a friend who was getting tested. One was male, the other transfemale.

\section{Question Responses}

Question 1: What does undetectable mean to you?

\section{Q1 Uninformed: $22 \%$ of total respondents.}

Slightly more than a fifth of participants $[\mathrm{n}=28]$ responded they did not know what 'undetectable' meant, or they gave a tautological answer (i.e. 'HIV virus undetectable'). The average age of this group was 36 years. Despite the tautology, about a third of these respondents indicated they understood this to mean the person was 'Still HIV+'.

One participant articulated nicely the thought process some people may use in interpreting what 'undetectable' means:

"In context of HIV undetectable sounds close to undetected -- perhaps undetectable means not found in the body or not present in the body." 28 y.o. white-Asian MSM

\section{Q1 Informed: $19 \%$ of respondents}

Less than a fifth of respondents indicated they were informed about what 'undetectable' means clinically. These respondents can be grouped into those who referenced viral load, those who referenced medication, and those who referenced both.

Over two thirds of Castro based participants fell into this category, but only $50 \%$ of Mission residents, and $44 \%$ of SoMa residents indicated they were informed. Of the most commonly reported zip codes, the Tenderloin and Glen Park gave the lowest showings, at less than a quarter falling into the informed group.

The average age of people who mentioned viral load was 40 years, and included 2 heterosexual men. The two positive people (MSM, TSM) were also in this group. Although respondents knew undetectable had to do with viral load, they indicated they weren't quite certain what the exact numbers were.

"Viral load less than certain threshold" 31 y.o. White MSM

"Means that a person has HIV but viral load is low enough to not be detected by test" 25 y.o. Black MSM

"HIV+ person whose viral load is too small to be measured" 37 y.o. White male

"The person has HIV infection but their viral load has been reduced to a point where testing shows their viral load is 'undetectable' according to lab results" 46 y.o white MSM

"People with +HIV but with virus reduced till the max neg" 42 y.o. Latino MSM

Many participants knew viral load pertained to measurements taken from the blood, but two people mentioned other body fluids:

"HIV+ person with no detectable HIV virus in blood or semen" 49 y.o. White married MSM

"HIV+ but 0 virus in body fluids/ blood" 30 y.o. Partnered Asian MSM 
A slightly younger set (average age 35 years) of 10 respondents, and all MSM, associated 'undetectable' with non-transmission of HIV. The following was a typical example:

"Someone who is infected with HIV virus, but are less likely to transmit the disease" 32 y.o. Hispanic MSM

An even younger group (average age 30 years), and again 10 respondents, all MSM, mentioned undetectable indicates someone taking medication. They evinced some variety in this understanding, however:

"It's a person who is HIV positive but is on treatment and viral load is undetectable to today's HIV tests" 31 y.o. Hispanic MSM

"Someone who reaches undetectable status is someone who is HIV positive but is treated so it takes longer to turn into AIDS" 19 y.o. Latino MSM

"It means the person is infected w/ HIV and they have a low count of the virus spread throughout body. Can be controlled and kept at low levels with ongoing treatment and medication." 29 y.o. Mixed race MSM

Finally, four people, split between two in their mid-late 20s, and two in their late 30s, all MSM, 3 white, 1 mixed race, gave answers which synthesised the above themes:

"It means someone is positive but on meds to reduce transmittability" 38 y.o. White MSM

"Patient has HIV under Dr care, taking prescription antiretrovirals/ under control and unlikely infecting others" 26 y.o. White MSM

\section{Q1 Misinformation: $36 \%$ of total respondents}

Over a third of participants evinced some degree of misinformation, ranging from the egregious to the problematic. The age group was on the younger side for the sample, at 32 years. In terms of racial breakdown, $42 \%$ of Asian respondents $[n=10]$ fell into this category, followed by $38 \%$ Latino $[n=6]$ and $35 \%$ of white respondents $[n=21]$. Black participants were least likely to be misinformed, with only a third of black respondents giving misinformation in their responses $[n=5]$. More than half the heterosexual respondents fell into this group $[\mathrm{n}=14=56 \%$, with the majority giving very misinformed answers.

Over $56 \%$ of Tenderloin residents indicated a degree of misinformation, with the Mission residents close behind at $45 \%$. Glen Park was also highly represented, with 38\%. SoMa and the Castro were lowest, at $11 \%$ and $21 \%$ of respondents giving misinformed answers, respectively.

Examples of some egregiously misinformed responses included:

"Meaning can't trace down the source of HIV infection due to a huge variety of sex intercourses [sic] or shared drug needles." 32 year old MSM of unknown race.

"Someone who has HIV, but don't know about it, never tested. Or tested, but result undetectable." 46 y.o. White heterosexual woman

"The patient has aids but not hiv, and is therefore undetectable, or vice versa?" 53 y.o. White, partnered MSM.

"It means somebody have STD and they don't know about it." 30 y.o. South Asian MSF.

Somewhat more benign, but still problematic included:

"Someone who has not tested" 27 y.o. Asian MSF 
Several respondents believed undetectable means asymptomatic or latent HIV, and at least two confused window period with undetectable.

"That you are infected but not showing symptoms or the infection is not currently strong enough to or the viral load/ antibody load is not high enough to return positive results on tests." 22 y.o. white heterosexual male. (Strikethrough in original response.)

"Virus dormant; quick test kits can't pick up certain virus strains" 30 y.o. Asian MSM

"Someone has the virus but is too soon to tell if infected" 23 y.o Latino MSM

By far the most common problematic thought was that the virus could not be detected by regular HIV tests, such as the finger stick test deployed at the testing site.

"I believe 'undetectable' means the blood that was tested was not able to give any results meaning the patient has to get blood drawn from arm and it will be sent to a lab" 19 y.o Black MSM

"Having HIV, but at a low enough viral count to be undetectable through regular HIV testing" 30 y.o. Latino MSM

"Means that you may carry the virus but it does not show on tests" 46 y.o. White transfemale (bisexual)

A few respondents mentioned other biometrics associated with HIV surveillance:

“Below 200 T-cell” 42 y.o. Filipino MSM

"Extremely low traces of HIV antibodies in bloodstream" 31 y.o. White MSM

One respondent indicated that the messaging of transmissibility was reaching him, but in a somewhat garbled fashion:

"I don't know. Obviously still infected, but tests negative. Unclear if still infectious." 31 y.o. White male

Overall, more than half of all respondents were either uninformed or misinformed about what 'undetectable' means. This was particularly notable among heterosexuals, Asians, and residents of the Tenderloin district. Of those who were educated about the topic, some understood the implications in terms of lowered risk to pass on or acquire HIV, while others took a more clinical test informed approach and cited viral load or medication adherence specifically.

\section{Question 2: How does someone know if they are undetectable?}

Q2 Uninformed: $26 \%$ of total respondents

About a quarter $(n=34)$ of respondents admitted to not knowing how someone knows their viral load is considered undetectable. The average age in this group was 34 years. While several ventured the generic response, 'by testing?', a few stated they didn't know and ventured other suggestions:

"I don't know (A doctor tells them?)" 27 y.o. Black man

"I suppose getting a more thorough blood sample or HIV test" 26 y.o. Black MSM w/ HIV+ boyfriend.

Q2 Informed: $56 \%$ of participants

A very high percentage $(n=55)$ of respondents, including one of the HIV + respondents, were confident people learned their status through a test, or through being told at a doctor visit. The average age was 36 years, and included over $40 \%(n=8)$ of the heteroseuxal men. Typical responses included:

"Through tests with their doctor or clinic" 60 y.o. White MSM 
“Check with the doctor" 42 y.o. Latino MSM

“Get tested and specific test for viral count" 49 y.o. Married white MSM

The most specific response was this one:

"They don't unless they take an RNA test" 36 y.o. Bisexual Asian male

Of the remaining informed responses, a significant number indicated both viral load testing and medication adherence were key to knowing one's undetectable status. With a similar age profile (35 years), this group was notable for being entirely MSMs.

"Regular (daily) meds and testing" 47 y.o. White MSM

"Someone knows they are undetectable after being diagnosed with HIV and regularly taking their HIV medications to keep viral load low." 30 y.o. White MSM

"Having a positive HIV test and then getting retested after being on HIV medication for a period of time" 30 y.o. Latino MSM

Q2 Misinformed: $17 \%$ of participants

By far the most common $(n=16)$ source of misinformation, accounting for almost three-quarters of the respondents in this category, was simple confusion between a viral load test and an HIV test:

"HIV test" 41 y.o. Asian MSM

The average age in this group was 34. In terms of categorization, if a respondent did not specify viral load (or RNA) testing, their response was placed in this category. However, this is not a benign confusion between HIV and viral load testing:

"Previous HIV test positive (confirmed), subsequent tests negative" 34 y.o. White MSF

"Results came back negative" 28. y.o. white MSM

Three additional people thought one could be undetectable at initial testing; the average age was 24 years:

"After having a finger blood test and the test comes back undetectable, a patient will be contacted, or they will do the test right then and there" 19 y.o. Black MSM

"They test positive or are informed by a sexual partner that they may have contracted the disease." 22 y.o. White MSF

An older subset ( $n=3$, average age 39 years) didn't believe one can ever know whether someone is undetectable, and yet they all indicated viral load was a component of knowing undetectable status (per question one). One person in this group indicated people must test regularly/ often to know. This group included one positive man:

"You don't, unless you tell them". 40 y.o. Asian male

Finally, one response was simply way off base:

"That's if someone takes part regularly in a group sexual involvement" 32 y.o. MSM, 94116 zip code.

Question 3: How long does someone remain undetectable?

Q3 Uniformed: $48 \%$ of total participants

Almost half $[n=62]$ the respondents were very frank about not knowing the answer to this question, which 
responses ranging from either 'no clue' to 'don't know'. The average age was slightly higher than the total study pool, at 37 years.

\section{Q3 Informed: $27 \%$ of participants}

About a quarter $[\mathrm{n}=35)$ of participants indicated that medication adherence -- including that the medication was still working -- was key to remaining undetectable, with an average age of 37 years for this group. With the exception of one heterosexual woman, these respondents were all MSM.

"As long as they take meds and get viral load checks" 43 y.o. White MSM

"As long as they are taking their meds; and get tested to redetermine their status" 40 y.o. Asian MSM

"As long as they are in treatment and viral load doesn't rebound -- only known through testing" 30 y.o. White MSM

A few respondents interpreted the question as if it asked how long someone remains undetectable if they become non-adherent:

"For a short period of time if they stop taking the medication" 42 y.o. Latino MSM

"Until the virus replicates to a problematic amount" 26 y.o. Black MSM

"Until they're tested as detectable and the immune system no longer can hold off the virus" 53 y.o. White MSM

\section{Q3 Misinformed: $25 \%$ of participants}

The remaining quarter gave misinformed responses, which ranged from sheer guesswork to clearly misguided understandings of the topic. The average age was slightly younger than the overall cohort, at 30 years, and included a third of the heteroseuxal repondents [ $\mathrm{n}=7$ male, 1 female], or nearly $40 \%$ of the straight men.

The guessed timeframes varied from the alarming 'permanent', 'for life', and 'continuously', through apparently randomly chosen years or months. One person said it is different for every person. A more articulate guess was the following:

"I believe after 1 month once the results come back a patient figures through an email or being contacted by phone to know when the results are back and the patient and doctor discuss the confidential information exclusively." 19 y.o. Black MSM

The idea of undetectable implying latent infection reappeared:

"I think until symptoms resurface or the virus is present when the sample blood is tested again" 28 y.o. Hispanic MSM

Some clearly misguided answers seemed to imply risk of reinfection, as if HIV were another STI that can be treated and return:

"Till they get infected" 27 y.o. Black male

“Until further sexual encounter" 28 y.o. White-Asian MSF

"Since they know how to protect themself" 16 y.o. Asian MSF

"Until they take measures to check themselves out" 18 y.o. White FSM

Question 4: How can we encourage you to continue testing every 3 - 6 months?

The final question differed from the foregoing in that it concerned testing, rather than beliefs about viral load. While $28 \%$ of the survey takers $[n=36$; average age 35 years] did not answer this question, the responses, when 
given, help inform consumer-driven policy and education endeavours.

About $18 \%$ [ $n=23]$ of the clientele, or a quarter of those who commented on this question, already tests regularly. The testing time frame ranged from every 45 days to every $6-9$ months. The average age for this group was 38 years, which is higher than the cohort as a whole. Generally people indicated they 'hate not knowing' or 'do it already'. One was on PrEP and so tests as part of his check ups.

The remaining $55 \%$ [ $n=71$, average age 34 years] offered multiple suggestions to encourage regular testing. Many people thought that technological outreach in the form of emails, text reminders, or ability to make online appointments would be useful for them.

A few focused on client experience, including one who suggested combining HIV and STI testing.

"Free cost, hassle-free, anonymous" [no demographics given -- note the anonymity]

"Probably shorter applications. Less fuss testing more personable testing." 46 y.o. Asian MSM

"Keep services open in the evenings and weekends" 38 y.o. Married Asian MSM

Others suggested advertisements, keeping testing free, and staying friendly.

"Increased outreach" 26 y.o. Black MSM

"By putting the word out there and creating awareness" 27 y.o. Black male

"Radio psa/ social media/ tv ads" 63 y.o. White MSM

"Publicity. As a man coming from another country, would be good to see a bit more of good publicity, and not necessarily about PrEP" 38 y.o. White male

"Reminders via email, phone etc. Flyers in the community." 27 y.o. White FSM living in SoMa

Several individuals stressed the need for education, with a particular split by age group in the MSM community:

"It's a catch 22: it's important to teach the importance" 26 y.o. White MSM

"Talk about the dangers of not being tested" 21 y.o. Latino MSM

"References to HIV centers and personal stories of individuals" 28 y.o. White MSM

"Free testing. Tell the truth about the ravages of AIDS to young gay men and the cost of PrEP for society." 49 y.o. White MSM

"Tell me the odds of getting HIV" 55 y.o. White male

And finally, one young man noted that stigma remains an obstacle to testing, giving a single word for his response:

"Destigmatize" 26 y.o Asian MSM

\section{Discussion and Significance}

Our study highlighted several trends, coalescing around sexual orientation, age, and zip code. The emerging picture of the uninformed tester is heterosexual, based in the Tenderloin, and Asian.

Sexual orientation and race. Overall, heterosexuals had the lowest levels of HIV education, and in some cases returned the most alarming responses. In terms of racial trends, Asians were surprisingly misinformed about what the term meant, and overall gave the most widely varying answers in terms of accuracy. Two factors may 
explain this: first, Asians were well represented among the small cohort of heterosexuals, who also had a high rate of misinformation. Second, in San Francisco encompass South Asian, East Asian, and Pacific Islander communities at various levels of English language proficiency and generations within the US. While one Asian respondent mentioned stigma against HIV, one response is not enough to determine how open discussing HIV is in Asian communities. Thus, the idea that stigma is particularly rife in Asian communities, based on the response of only a single (otherwise unspecified) Asian-identified participant who suggested 'destigmatize', seems overdetermined.

MSMs, as a whole, seem fairly well versed in what 'undetectable' means, with a sizable group only understanding that 'undetectable' means 'less likely to transmit HIV,' indicative of what messaging is going through. However, without the follow-up details of how 'undetectable' is known and maintained as a biological status, this understanding can be manipulated by misinformation -- e.g. 'undetectable means for life'. Overall, Black respondents were the least likely to be misinformed about what 'undetectable' means, which lines up with other studies correcting misinformation and stereotypes regarding the sex lives and level of sexual education among Black MSMs. [13, 14, 15]

Age. Age differences in how MSMs responded suggest a desire to transmit and learn the cultural history of HIV and AIDS. Interestingly, the responses evince a generational divide that roughly aligns with the state of HIV treatments when the men were in their 20s: men older than 47 years recall the era of AIDS and the cocktails that were given, the 'ravages' as one participant called it. This more mature demographic was most cautious, if not altogether uninformed, when approaching the idea of 'undetectable', and more invested in biomedical surveillance as a source of knowledge. Men in their 30s came of age after protease inhibitors found success and ARVs were introduced; they may have heard personal stories, and seem oriented towards medication and viral load surveillance both. In contrast, younger men in their twenties are asking for stories, having come of age in the era of PrEP and HIV as a chronic illness. They were most likely to reference medication, rather than viral load, when defining what undetectable means. They were also less likely to understand how an undetectable status was determined, and misinformed about how long such a finding is valid.

Zip Code. Several San Francisco neighborhoods are known for being areas of high transmission of HIV and lower suppression. $[8,12]$ Among the lowest suppressed zip codes is the Tenderloin, which in our study corresponds with the zip code associated with the highest percentage of misinformation about the meaning and implications of 'undetectable' abound.

Taking account of Table 3 and Table 5 together, the Tenderloin, SoMa, and Glen Park areas need more accurate information about what 'undetectable' means. The Castro and Nob Hill, on the other hand, seems fairly well informed about what 'undetectable' implies, but remarkably uninformed about how someone knows they are undetectable. This may be because people testing negative for HIV don't understand what the spectrum of HIV care entails, part of which is regular viral load testing. Likewise, Castro-based respondents also seem uninformed about how long such a finding is valid. The Mission, in contrast, seems overall well informed.

In terms of hypothesizing why some neighborhoods have a higher degree of misinformation, if we consider neighborhoods as individual entities with a treatment history of their own at the geographic and population level, Glen Park can be considered an outlier neighborhood in terms of the length of treatment time at a community level, while the Castro has been 'in treatment' since the inception of the epidemic. In other words, Glen Park is akin to an 'ARV naive' patient, but at the neighborhood level, with fewer dispensaries and pharmacies dedicated to ARV provisioning, fewer informational pamphlets available and for a shorter period of time, and a lower density of people living with HIV in comparison to the Castro. In other words, Glen Park has not yet had the historical experience of treatment long enough to reach a threshold of information saturation and network communication within the neighborhood at the individual level.

Explaining why the Tenderloin is among the more informed areas when it comes to knowing an undetectable status and how long it lasts, in contrast with the degree of misinformation about what it means, is more difficult to explain using the above model of neighborhoods as 'in care' or 'treatment naive'. It may be that the Tenderloin, as an area of relatively broader biomedical and public health surveillance, is more likely to orient residents towards reliance on officials to determine labels. Understanding what those labels mean, however, is another matter -- and perhaps for the residents on an individual level, it doesn't matter; being seen at regular intervals may be more important. 


\begin{tabular}{|c|c|c|c|c|c|c|}
\hline Zip & Quest. & Uninformed & Informed & Misinformed & Total(s) & $\%$ Informed \\
\hline 94102 & $q 1$ & 2 & 2 & 5 & 9 & $22 \%$ \\
\hline \multirow[t]{2}{*}{ 'Tenderloin' } & $q 2$ & 4 & 5 & 0 & 9 & $55 \%$ \\
\hline & $q 3$ & 7 & 1 & 1 & 9 & $11 \%$ \\
\hline $30 \%$ informed & $t$ & 13 & 8 & 6 & & \\
\hline 94103 & $q 1$ & 4 & 4 & 1 & 9 & $44 \%$ \\
\hline \multirow[t]{2}{*}{ 'SoMa' } & $q 2$ & 4 & 3 & 2 & 9 & $33 \%$ \\
\hline & $q 3$ & 5 & 3 & 1 & 9 & $33 \%$ \\
\hline $37 \%$ informed & $t$ & 13 & 10 & 4 & & \\
\hline $94109 *$ & $q 1$ & 1 & 2 & 3 & 6 & $33 \%$ \\
\hline \multirow[t]{2}{*}{ 'Nob Hill' } & $q 2$ & 0 & 5 & 1 & 6 & $83 \%$ \\
\hline & $q 3$ & 0 & 4 & 2 & 6 & $67 \%$ \\
\hline $61 \%$ informed & $t$ & 1 & 11 & 6 & & \\
\hline 94110 & $q 1$ & 0 & 6 & 5 & 11 & $55 \%$ \\
\hline \multirow[t]{2}{*}{ 'Mission' } & $q 2$ & 2 & 8 & 1 & 11 & $73 \%$ \\
\hline & $q 3$ & 3 & 5 & 3 & 11 & $45 \%$ \\
\hline $58 \%$ informed & $t$ & 5 & 19 & 9 & & \\
\hline 94114 & $q 1$ & 0 & 10 & 3 & 13 & $77 \%$ \\
\hline \multirow[t]{2}{*}{ 'Castro' } & $q 2$ & 5 & 7 & 1 & 13 & $54 \%$ \\
\hline & $q 3$ & 9 & 3 & 1 & 13 & $23 \%$ \\
\hline $51 \%$ informed & $t$ & 14 & 20 & 5 & & \\
\hline $94117^{*}$ & $q 1$ & 2 & 3 & 2 & 7 & $43 \%$ \\
\hline 'Haight/ Cole & $q 2$ & 1 & 5 & 1 & 7 & $71 \%$ \\
\hline Valley' & $q 3$ & 2 & 3 & 2 & 7 & $43 \%$ \\
\hline $52 \%$ informed & $t$ & 5 & 11 & 5 & & \\
\hline 94131 & $q 1$ & 3 & 2 & 3 & 8 & $25 \%$ \\
\hline \multirow[t]{2}{*}{ 'Glen Park' } & $q 2$ & 1 & 5 & 2 & 8 & $63 \%$ \\
\hline & $q 3$ & 3 & 3 & 2 & 8 & $38 \%$ \\
\hline $42 \%$ informed & $t$ & 7 & 10 & 7 & & \\
\hline
\end{tabular}

* Zip Code significant because of Question 2 responses

Table 3: Zip Codes Ranked from Least Informed to Most Informed

\begin{tabular}{cccc} 
Question 1 & Question 2 & Question 3 & Overall \% \\
\hline $94102(22 \%)$ & $94103(33 \%)$ & $94102(11 \%)$ & $94102(30 \%)$ \\
$94131(25 \%)$ & $94114(54 \%)$ & $94114(23 \%)$ & $94103(37 \%)$ \\
$94109(33 \%)$ & $94102(55 \%)$ & $94103(33 \%)$ & $94131(42 \%)$ \\
$94117(43 \%)$ & $94131(63 \%)$ & $94131(38 \%)$ & $94114(51 \%)$ \\
$94103(44 \%)$ & $94117(71 \%)$ & $94117(43 \%)$ & $94117(52 \%)$ \\
$94110(53 \%)$ & $94110(73 \%)$ & $94110(45 \%)$ & $94110(58 \%)$
\end{tabular}




$94114(77 \%) \quad 94109(83 \%) \quad 94109(67 \%) \quad 9410961 \%)$

Table 4: Zip Codes Ranked from Most Misinformed to Least Misinformed

\begin{tabular}{llll}
\hline Question 1 & Question 2 & Question 3 & Overall \% \\
\hline $94102(56 \%)$ & $94114(77 \%)$ & $94114(77 \%)$ & $94109(33 \%)$ \\
$94109(50 \%)$ & $94131(25 \%)$ & $94109(33 \%)$ & $94131(29 \%)$ \\
$94110(45 \%)$ & $94103(22 \%)$ & $94117(29 \%)$ & $94110(27 \%)$ \\
$94131(38 \%)$ & $94109(17 \%)$ & $94110(27 \%)$ & $94117(24 \%)$ \\
$94117(29 \%)$ & $94117(14 \%)$ & $94131(25 \%)$ & $94102(22 \%)$ \\
$94114(23 \%)$ & $94110(9 \%)$ & $94102(11 \%)$ & $94103(15 \%)$ \\
$94103(11 \%)$ & $94102(0 \%)$ & $94103(11 \%)$ & $94114(13 \%)$
\end{tabular}

Table 5: Zip Codes Ranked from Most Uninformed to Least Uninformed

\begin{tabular}{llll}
\hline Question 1 & Question 2 & Question 3 & Overall \% \\
\hline $94103(44 \%)$ & $94102(44 \%)$ & $94102(78 \%)$ & $94102(48 \%)$ \\
$94131(38 \%)$ & $94103(44 \%)$ & $94114(69 \%)$ & $94103(48 \%)$ \\
$94117(29 \%)$ & $94114(38 \%)$ & $94103(56 \%)$ & $94114(36 \%)$ \\
$94102(22 \%)$ & $94110(18 \%)$ & $94131(38 \%)$ & $94131(29 \%)$ \\
$94109(17 \%)$ & $94117(14 \%)$ & $94117(29 \%)$ & $94117(24 \%)$ \\
$94110(0 \%)$ & $94131(13 \%)$ & $94110(27 \%)$ & $94110(15 \%)$ \\
$94114(0 \%)$ & $94109(0 \%)$ & $94109(0 \%)$ & $94109(6 \%)$
\end{tabular}

Additional findings. Some participants were quite articulate about the thought processes behind their answers to the survey questions. This includes thinking of the direct implications of terminology used ('undetectable $=$ not found'), but also personal insight into the assumption that knowing someone's HIV status and viral load entails an implicit degree of trust and open communication.

A very common confusion among all participants concerned the multiplicity of tests and what they tested for. Some respondents believed that a blood test would indicate viral load counts for both semen and body fluids. In terms of knowing what is tested for, many participants believed HIV antibody and p24 antigen tests could determine viral load, or that RNA based viral load testing would indicate levels of antibody or T-cells circulating in the body.

Clients indicated they are very open to electronic reminders to come in for regular testing. They are noted this is a good, though by no means only, means to deliver up-to-date information about the latest HIV treatments and findings.

Limits of the Study. Survey limitations rest on it being a single site, self selected, almost entirely HIV-negative (at time of testing) population. While the sample size was relatively small, it was generally reflective of the demographic who regularly test at the clinic. The demographic information did not distinguish Asians by community, and anecdotal evidence from test counselors indicates differing levels of HIV education (and anxiety) based on community of origin.

Given the small set of women, we decided to nonetheless include them in the sample simply to increase the number of heterosexuals represented. In a disease demographic characterised not by differences in risk by gender, but by sexual orientation, it is important to pay attention to both male and female heterosexuals. Given the degree of misinformation consistently exhibited by our very small number of heterosexual respondents, evidence suggests this population has been overlooked in efforts to educate people on, and consequently destigmatize, HIV. [16]

The use of zip codes to approximate neighborhoods also has its drawbacks, as some zip codes encompass what are organically up to three different neighborhoods. These neighborhoods at times include testing sites which 
serve a somewhat different demographic than those who come into our testing site. Nevertheless, as a proxy for demographic trends, they do give a broad geo-cultural idea of ongoing developments.

Finally, although space was provided for somewhat elaborate responses to the questions asked, the presence of vague responses warrants deeper interviewing via focus groups.

\section{Suggestions}

By Zip Code. Public outreach in the Tenderloin, the associated areas of SoMa (as suggested by a participant), and Nob Hill seem warranted. Given the stratification of surveillance in these areas, however, such outreach may not need to be targeted to specific groups within the zip code so much as the neighborhood as a whole. That is, outreach may already be successful among sub-groups involved in various treatment programs, but may not be reaching the Tenderloin-based population who test at the survey site if they fall outside those targeted groups. Glen Park is an emerging additional neighborhood to target with informational campaigns.

While many in the Castro district already associate medication with undetectable status, it is important to emphasise the role of ongoing ARV adherence, particularly among older MSMs (in terms of this study, older than the mean cohort age, thus late 30 s and up). Among younger people (20s and early 30 s) in this neighborhood, what needs emphasis is the fact that 'undetectable' isn't a 'permanent' diagnosis.

Since data indicates that participants residing closer to Castro clinics are better informed, further inquiry into the sources of information is warranted, especially when the question of focusing informational resources is raised. Potential sources of information already include local media outlets, Gloss, B.A.R., and personal networks versus established clinics frequented by residents of this neighborhood (e.g. Strut, AHP, API).

By emerging profile of the uninformed. The Tenderloin has emerged as a geographical area experiencing an information gap, but so has the demographic of heterosexuals and Asians. One way to address the racial disparity in San Francisco is to further parse demographics more closely: do differences emerge by national origin, number of generations in the US, or English language proficiency? Since Asians are typically regarded as a highly educated demographic, is the absence of sexual health information locatable to schools or to percentage of foreign born (and thus educated) participants?

Educational outreach regarding how long someone remains undetectable is necessary. This is especially the case among heterosexuals, who evince less knowledge on the topic than MSMs. Likewise, for both MSMs and heterosexuals, differentiating viral load tests from HIV tests (and T-cell count from viral load) would be useful, though this is a 'completing the education' step by informing people about what medical care happens after an HIV diagnosis. Not only would such education go some way to alleviating the idea that an HIV+ person won't test positive once he or she has lowered the viral load to 'undetectable', it may also serve to decrease stigma against the positive community, and fear of getting tested in the first place. These needs can be met through continuing advertising campaigns for testing centers and for educational efforts aimed at destigmatizing testing and treatment.

For further study. Two areas for theoretical study and modelling came up. A modelling study could, in theory, run statistical analyses of transmission patterns based on behavioural predictions extrapolated from beliefs about 'undetectable' and 'treatment as prevention'. For example, if everyone in a community of 100,000 shared a particular single belief such as 'undetectable = cured' or 'undetectable = permanent', what would transmission patterns look like? Running such 'game theory' type analyses would then help prioritize which misinformed and uninformed beliefs are most in need of addressing.

The fact that multiple participants seem to believe 'undetectable' means a 'dormant' virus, is a warning sign about a potentially dangerous gap in people's understandings of HIV and other chronic or transmissible diseases. Thus, in a medical anthropology vein, focus groups could untangle what people mean when they say HIV is dormant, or the symptoms are latent. How this interacts with biometrics like viral load, T-cells, and antibodies -- or how these each impact transmissibility -- could then be explored. Likewise, it might be useful to investigate the range of knowledge people living with HIV have about the term 'undetectable', and not only how they deploy it socially and in clinical contexts.

Patient-driven suggestions. Educating clients on the different types of tests (HIV, RNA/ Viral Load, etc) raises the larger question of what degree of specificity should a counselor aim at giving clients who come in to test. 
While it may seem that imparting a minimum, simple level of knowledge is best, participant responses to how we can encourage regular testing suggest that both younger and older MSMs desire this knowledge. Relatedly, but not generally the focus of educational campaigns focused on transmission, young MSMs have requested access to more personal stories of living with HIV, of surviving the era of AIDS, and of a fuller history of the community and its defining epidemic. This is a consumer driven demand for health information that coincides with the stake public health officials have in ending the epidemic by filling out the larger contextual history of how we got to 'Getting to Zero' and the San Francisco model of HIV care.

\section{References:}

[1] Wilson, D; Law, M; Grulich, A; Cooper, D; Kaldor, J (2008). "Relation between HIV viral load and infectiousness: a model-based analysis.” The Lancet, Volume 372, Issue 9635, 26 July-1 August 2008, Pages 314-320.

[2] Attia, Suzannaa; Egger, Matthiasa; Müller, Monikaa; Zwahlen, Marcela; Low, Nicolaa (2009). Sexual transmission of HIV according to viral load and antiretroviral therapy: systematic review and meta-analysis. AIDS: 17 July 2009 - Volume 23 - Issue 11 - p 1397-1404 doi: 10.1097/QAD.0b013e32832b7dca

[3] Holt, Martin; Lea, Toby; Mao, Limin; Zablotska, Iryna; Prestage, Garrett; de Wit, John (2015). Brief Report: HIV Prevention by Australian Gay and Bisexual Men With Casual Partners: The Emergence of Undetectable Viral Load as One of a Range of Risk Reduction Strategies. JAIDS Journal of Acquired Immune Deficiency Syndromes: 15 December 2015 - Volume 70 - Issue 5 - p 545-548. doi: 10.1097/QAI.0000000000000787.

[4] E. Kuhn, A. Potthoff, H. Dirks, R. Borgmann, S. Esser, N. Scherbaum, N.H. Brockmeyer, A. Skaletz-Rorowski (2016). Viral load strategy: impact on risk behaviour and serocommunication of men who have sex with men in specialized care. JEuroAacadDermVenereology, Volume 30, Issue 9, September 2016. Pages 1561-1566 doi: $10.1111 / \mathrm{jdv} .13672$

[5] Seth C. Kalichman, Chauncey Cherry, Moira O. Kalichman, Christopher Washington, Tamar Grebler, Ginger Hoyt, Cindy Merely, Brandi Welles (2015). Sexual Behaviors and Transmission Risks Among People Living with HIV: Beliefs, Perceptions, and Challenges to Using Treatments as Prevention. Archives of Sexual Behavior. August 2016, Volume 45, Issue 6, pp 1421-1430

[6] Newcomb, Michael E; Mongrella, Melissa C; Weis, Benjamin; McMillen, Samuel J; Mustanski, Brian (2016). Partner Disclosure of PrEP Use and Undetectable Viral Load on Geosocial Networking Apps: Frequency of Disclosure and Decisions About Condomless Sex. JAIDS Journal of Acquired Immune Deficiency Syndromes: 1 February 2016 - Volume 71 - Issue 2 - p 200-206. doi: 10.1097/QAI.0000000000000819

[7] Guigayoma, John; Chen, Yea-Hung; Snowden, Jonathan; Santos, Glenn-Milo; Hecht, Jennifer; Raymond, H. Fisher (2014). Self-Perceived Viral Load and Sexual Risk Behavior Among Known HIV-Positive MSM in San Francisco, 2014. JAIDS Journal of Acquired Immune Deficiency Syndromes: 1 July 2017 - Volume 75 - Issue p S309-S315. doi: 10.1097/QAI.0000000000001405

[8] Highleyman, L (2016). "San Francisco reports new low in HIV infections and faster treatment, but disparities remain" AIDSmap.

http://www.aidsmap.com/San-Francisco-reports-new-low-in-HIV-infections-and-faster-treatment-but-disparities -remain/page/3082266/

[9] Das M, Chu PL, Santos G-M, Scheer S, Vittinghoff E, McFarland W, et al. (2010) Decreases in Community Viral Load Are Accompanied by Reductions in New HIV Infections in San Francisco. PLoS ONE 5(6): e11068. https://doi.org/10.1371/journal.pone.0011068

[10] Miller, C; Powers, K; Smith, MK; Cohen, M (2013). Community viral load as a measure for assessment of HIV treatment as prevention. Volume 13, Issue 5, May 2013, Pages 459-464.

https://doi.org/10.1016/S1473-3099(12)70314-6 
[11] Infectiousness by sexual network thresholds:

http://mpe.dimacs.rutgers.edu/wp-content/uploads/2013/06/Small.change.gif

[12] SF DPH Population Health Division (September 2016). "HIV Epidemiology Annual Report 2015" https://www.sfdph.org/dph/files/reports/RptsHIVAIDS/AnnualReport2015-20160831.pdf -- p75ff, with maps.

[13] Maulsby, C., Millett, G., Lindsey, K. et al. (2014). "HIV Among Black Men Who Have Sex with Men (MSM) in the United States: A Review of the Literature." AIDS Behav (2014) 18: 10.

https://doi.org/10.1007/s10461-013-0476-2

[14] Millett GA, Flores SA, Peterson JL, Bakeman R. "Explaining disparities in HIV infection among black and white men who have sex with men: a meta-analysis of HIV risk behaviors." AIDS. 2007;21(15):2083-91.

[15] Manya Magnus, Irene Kuo, Gregory Phillips II, Katharine Shelley, Anthony Rawls, Luz Montanez, James Peterson, Tiffany West-Ojo, Shannon Hader, and Alan E. Greenberg (2010). "Elevated HIV Prevalence Despite Lower Rates of Sexual Risk Behaviors Among Black Men in the District of Columbia Who Have Sex with Men.” AIDS Patient Care and STDs. October 2010, 24(10): 615-622. https://doi.org/10.1089/apc.2010.0111 --

[16] Henry, T (2010). Heterosexual poor at higher risk for AIDS. CNN Health Plus. http://thechart.blogs.cnn.com/2010/07/19/heterosexual-poor-at-higher-risk-for-aids/

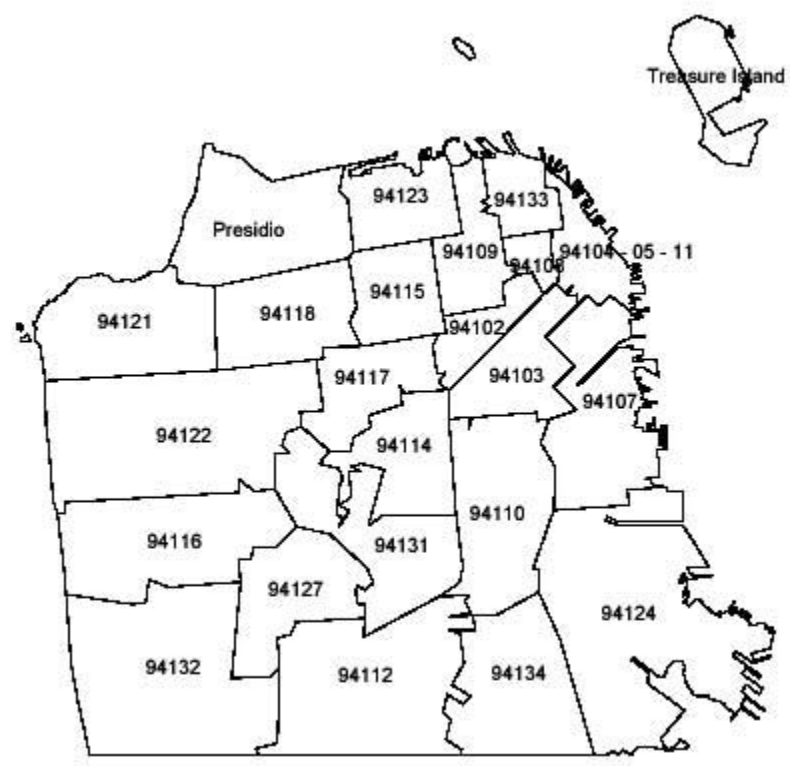

San Francisco mapped by Zip Codes. Source: http://www.healthysf.org/bdi/outcomes/images/zip-map.jpg 